\section{The role of JAK2 abnormalities in hematologic neoplasms}

\author{
Mohammed K. Alabdulaali \\ Blood Diseases Center, King Fahad Hofuf \\ Hospital, Hassa, Saudi Arabia
}

\begin{abstract}
In 2005, an activating mutation in the Janus kinase 2 (JAK2) was identified in a significant proportion of patients with myeloproliferative neoplasms, mainly polycythemia vera, essential thrombocythemia and primary myelofibrosis. Many types of mutations in the JAK-STAT pathway have been identified, the majority are related to JAK2. Currently JAK2 mutations are important in the area of diagnosis of myeloid neoplasms, but its role beyond the confirmation of clonality is growing and widening our knowledge about these disorders. In addition to that, clinical trials to target JAK2-STAT pathway will widen our knowledge and hopefully will offer more therapeutic options. In this review, we will discuss the role of JAK2 abnormalities in the pathogenesis, diagnosis, classification, severity and management of hematologic neoplasms.
\end{abstract}

\section{Introduction}

For decades the diagnosis, classification and management of hematologic neoplasms was based on the clinico-pathological features of these disorders. Major progress in both the therapeutics and our understanding of these diseases did not occur until 1960 when Philadelphia chromosome was found in bone marrow cells of patients with chronic myelogenous leukemia (CML), followed by the identification of the molecular defect by the fusion of $B C R$ and $A B L$ genes in 1982. Fifteen years later imatinib, an ABL tyrosine kinase (TYK) inhibitor, was developed in the late 1990s. Nowadays the diagnosis of CML is dependent on the identification of $\mathrm{t}(9 ; 22)(\mathrm{q} 34 ; \mathrm{q} 11)$ or $B C R / A B L$ fusion gene and imatinib is one of its first line therapeutics. Other hematologic neoplasms are tracking the footprints of CML. $B C R / A B L$ negative myeloproliferative neoplasms (MPNs), particularly the classical polycythemia vera (PV), essential thrombocythemia (ET) and primary myelofibrosis (PMF), are currently drawing the attention of many scientists in the fields of hematology, oncology, pathology, genetics and pharmacology, after the identification of Janus kinase 2 (JAK2) mutation in a significant number of patients diagnosed for these disorders in $2005 .{ }^{1}$
In this article, our aim is to review the role of JAK2 abnormalities in the pathogenesis, diagnosis, classification, severity and management of hematologic neoplasms.

\section{Classification of myeloid neo- plasms}

As JAK2 abnormalities are mainly identified in myeloid neoplasms, we will summarize the current classification scheme for these disorders. In general they are divided into 3 major groups: acute leukemia, chronic leukemia and myelodysplastic syndrome. Chronic leukemia can be sub-divided into $B C R / A B L$ positive or negative, those $B C R / A B L$ positive will be labeled as CML regardless of their clinicopathological features unless it is presenting as acute leukemia, while those $B C R / A B L$ negative will be divided further into classical MPNs, non-classical MPNs with or without dysplasia, and myelodysplastic syndrome (MDS) (Figure 1). The diagnosis and classification of these disorders are based on peripheral blood counts, blast percentage, type of myelosis, presence of significant dysplasia, extent of fibrosis, clinical features, biochemistry and most importantly genetics. ${ }^{2-4}$

JAK2 abnormalities are not only associated with most of the classical myeloid neoplasms but they are also seen in association with other myeloid neoplasms except $B C R / A B L$ positive CML and acute lymphoid leukemia (ALL) where it is only rarely reported as we will see later on.

One of the interesting things regarding the clinical features of myeloid neoplasms is their tendency to transform to acute leukemia; the classical MPNs, beside their pre-leukemic behavior, progress and regress to each other (Figure 2)..$^{3-5}$

So, why does an abnormal gene give rise to different disorders? Why do classical MPNs progress and regress? What are the roles of $J A K 2$ abnormalities in the pathogenesis of hematologic neoplasms?

\section{JAK family}

Janus kinase is a family of intracellular nonreceptor tyrosine kinases that transduce cytokine-mediated signals. At present, it consists of 4 members: JAK1, JAK2, JAK3 and TYK2. ${ }^{6}$

Janus kinases was named after Janus or Ianus who in Roman mythology was believed to be the God of gates, beginnings and endings. He was imagined as having two faces or heads facing in opposite directions. ${ }^{?}$

Indeed, Janus kinases are located just
Correspondence: Mohammed K. Alabdulaali, Blood Diseases Center, King Fahad Hofuf Hospital, P.O. Box: 1 31982, Hassa, Saudi Arabia E-mail: drmohka@hotmail.com

Key words: JAK2, myeloproliferative neoplasms, leukemia.

Acknowledgment: I would like to thank Dr. Khalid M. Alayed, King Khalid University Hospital, for his helpful discussion and enlightening views.

Received for publication: 3 May 2009.

Revision received: 3 June 2009.

Accepted for publication: 24 June 2009.

This work is licensed under a Creative Commons Attribution 3.0 License (by-nc 3.0)

(C) Copyright M.K. Alabdulaali et al., 2009

Licensee PAGEPress, Italy

Hematology Reviews 2009; 1:e10

doi:10.4081/hr.2009.e10

beneath the cellular receptors to control the signal transmission downstream and have seven domains, two of which are structurally similar. One of these (JH1) is an activating domain while the other (JH2) seems to exert an inhibitory effect (Figure 3).

Upon ligand binding to its specific receptor, JAK protein will be activated and it will then phosphorylate the downstream signaling molecules like STATs, which will be actively transported to the nucleus where it will activate transcription factors (Figure 4).

Abnormalities in JAK1 have been reported in ALL of mainly T cell type where it is found in nearly $20 \%$ of the cases, ${ }^{8} J A K 2$ is found in myeloid neoplasms and rarely rearranged in ALL, ${ }^{6}$ JAK3 have been reported in more than $50 \%$ of transient abnormal myelopoiesis in Down syndrome patients, acute myeloid leukemia (AML) megakaryoblastic type (M7) and in some cases of severe combined immune deficiency (SCID), ${ }^{9-11}$ and TYK2 might have a role in lymphoid neoplasms and natural killer cell functional defects. ${ }^{12}$

\section{JAK2}

$J A K 2$ was mapped on the short arm of chromosome 9p24 in 1992 by Pritchard and his colleagues, ${ }^{13}$ It has $140 \mathrm{~kb}$ spanning 25 exons to form 1132 aminoacid JAK2 protein. ${ }^{14}$ It works as a signaling molecule for many cytokines including: INF- $\gamma,{ }^{15}$ erythropoietin (EP0) ${ }^{16}$ prolactin, ${ }^{17}$ thrombopoietin (TMP), G-CSF, GM-CSF ${ }^{18}$ and IL-3 ${ }^{19}$ via activating many signaling pathways like: MAPK, PI $3,{ }^{16}$ ERK $^{20}$ and STATs. ${ }^{14}$ PRV1 (CD177) and NF-E2 
also appear to be activated and overexpressed by JAK2. ${ }^{6}$ One of the most important pathways that are activated by JAK2 is STAT5 followed by activation of $B C L-X L$ and finally upregulation of $B C L 2$ where the cell will gain a survival advantage. ${ }^{6}$

\section{Types of JAK2 abnormalities and pathogenesis}

In general we can divide these into 4 categories: ${ }^{4,21-25}$

A. Rearrangements

JAK2 can be rearranged to:

- TEL/ETV6: $\mathrm{t}(9 ; 12)$ (p24;p13) reported in some CML like MPNs and T-cell ALL;

- BCR: $\mathrm{t}(9 ; 22)$ (p24;q11.2) reported in some CML like MPNs;

- PCM1: t(8;9) (p22;p24) reported in some MPNs, AML and ALL;

- NF-E2: $\operatorname{der}(9) \mathrm{t}(9 ; 12)$ (p24;q13) reported in some cases of MDS.

B. Point mutations

- V617F G > T at nucleotide 1849 on exon14, reported in mainly the classical MPNs;

- T875N reported in AML (M7);

- R683G and less frequently other R683 point mutations reported in 18-28\% of ALL in Down syndrome patients and $10 \%$ of a high-risk cohort of childhood ALL in patients without Down syndrome.

C. Deletions/Insertions

- Exon12: there are more than eight reported mutations including deletions and insertions in 538 to 543 codons report ed in 4\% of PV cases;

- IREED del which is a five amino acids deletion in JH2 pseudokinase domain reported in B-cell ALL in Down syndrome patients.

D. Numerical

- It can present as trisomy (+9) or be overexpressed due to amplification.

The majority of these abnormalities are affecting the JH2 domain leading to loss of inhibitory effects on JH1 domain, hence the later will be auto-activated. Suppressors of cytokine signaling (SOCS) 1 and 3 are negative regulators for JAK2 kinase, these suppressors will also be phosphorylated and stabilized by the hyperactive tyrosine kinase. ${ }^{26}$ SOCS3 promoter methylation is another mechanism that is found in a group of patients. ${ }^{27}$

It is reported that $5-10 \%$ of MPN patients have at least one relative who is affected by this disorder, and in familial MPNs the risk of developing these disorders increases 6 -fold. ${ }^{6,28}$ Recent studies suggest that particular single nucleotide polymorphisms (SNP) are associated with a higher risk of developing JAK2 V617F mutation. Out of about 659 SNPs, rs 10974944 and $\mathrm{rs} 12343867$ are reported in $77 \%$ and $85 \%$ in association with $J A K 2 \mathrm{~V} 617 \mathrm{~F}$ mutation

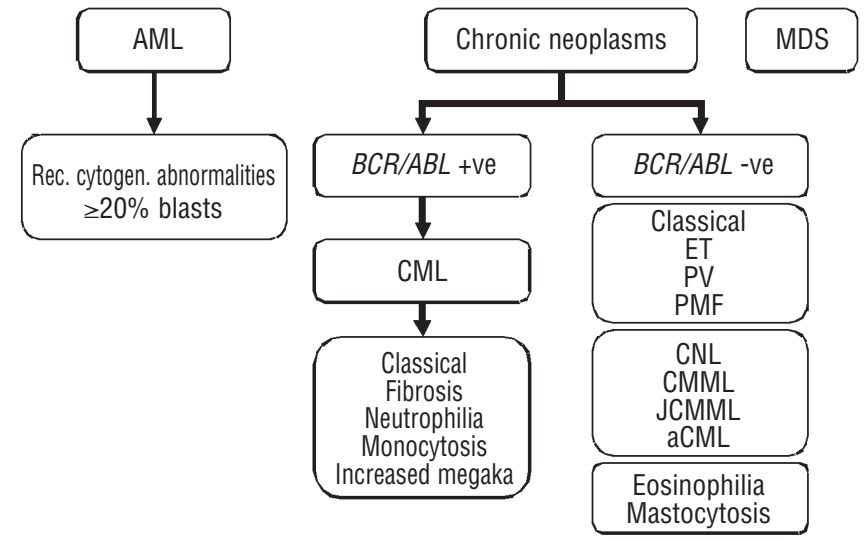

Figure 1. The current classification of myeloid neoplasm. CNL: chronic neutrophilic leukemia, CMML: chronic myelomonocytic $1 \mathrm{e} \mathrm{u} \mathrm{k} \mathrm{e} \mathrm{m} \mathrm{i} \mathrm{a} \mathrm{,}$ JCMML: juvenile myelomonocytic leukemia, aCML: atypical chronic m yelogenous leukemia.

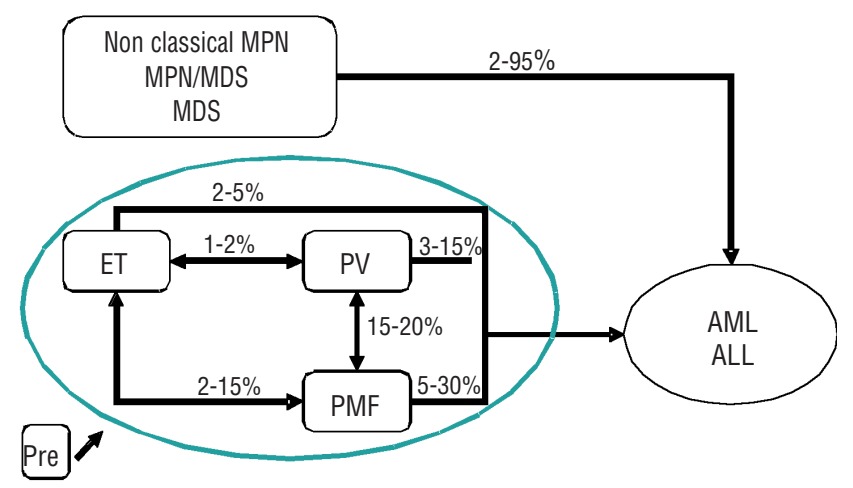

Figure 2. The rate of progression of chronic myeloid neoplasms to leukemic phase. Pre: pre-fibrotic stage of PMF and un expla in ed thrombotic events prior to the development of MPN.

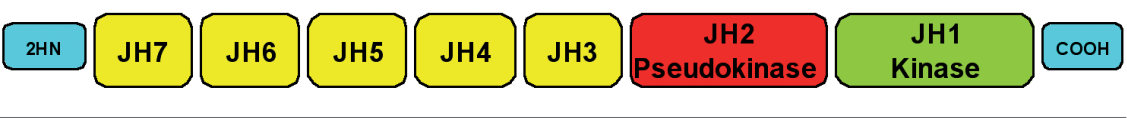

Figure 3. The structure of Janus kinases

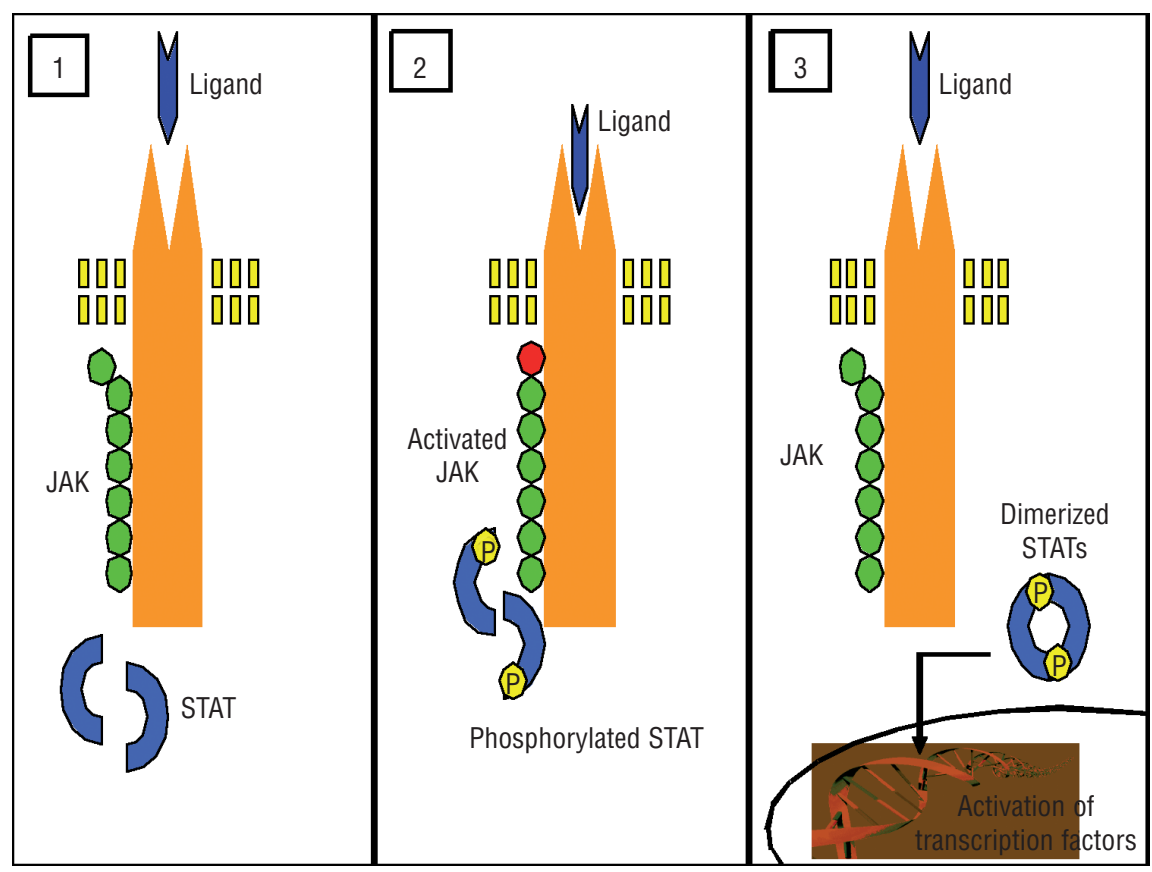

Figure 4. Steps of JAK-STAT pathway. 
respectively, with a significant difference if compared to wild-type $J A K 2{ }^{29}$

\section{Prevalence of JAK2 abnormali- ties in hematologic neoplasms}

JAK2 V617F mutation is reported only in myeloid neoplasms with a high frequency in PV, ET, PMF and refractory anemia with ring sideroblast and thrombocytosis (RARS-t). It is rarely reported in CML, ${ }^{30}$ but not in ALL or molecularly characterized eosinophilic neoplasms and mastocytosis, i.e. those with abnormalities in PDGFRA, PDGFRB, FGFR1 or KIT. JAK2 rearrangements are seen in some cases of AML, atypical CML and ALL, while exon 12 mutations are only reported in PV (Table 1)., ${ }^{3,431,32}$

\section{Screening and quantification tests}

Detecting JAK2 mutations, determination of hetero-or homozygosity, and the allele burden, i.e. the ratio of the mutant allele to the wildtype, are the aims of carrying out molecular studies. Variable screening techniques are available and these are basically utilizing direct sequencing, allele specific polymerase chain reaction (PCR) analysis or ultra sensitive PCR techniques with variable sensitivities that can detect mutant gene at the levels of $20 \%, 3 \%$ and $0.01 \%$, respectively. However, quantification techniques are preferred in order to estimate the mutant allele burden, to monitor the disease course and effect of therapeutics. Peripheral blood and bone marrow samples, frozen plasma and paraffin-embedded trephine bone marrow biopsies can all be used. ${ }^{2,33}$ Eric Lippert and his colleagues studied the concordance of assays designed for the quantification of $J A K 2 \mathrm{~V} 617 \mathrm{~F}$ and reported the highest sensitivity by using Taqman allele specific PCR with reverse and forward primers with detection sensitivity up to $0.2 \%$ and $0.15 \%$, respectively. But they found these techniques laborious, having false positive results and they were, as expected, capable of detecting only the mutation of interest. On the other hand, they found pyrosequencing and direct DNA sequencing to be the least sensitive, limited to the level of $2 \%$ and $5 \%$, respectively, but still having the advantage of detecting new mutations. ${ }^{34}$ It has recently been suggested that immunoprecipitations and Western blotting to test for SOCS3 tyrosine phosphorylation may be a novel bio-marker of MPNs resulting from a $J A K 2$ mutation and a potential reporter of effective JAK2 inhibitor therapy currently in clinical development. ${ }^{26}$

\section{The roles of JAK2 abnormali- ties in hematologic neoplasms}

\section{Confirmation of clonality}

The long list of congenital, secondary or reactive causes for cytosis, cytopenia and dysplasia is creating difficulties in labeling cases for neoplastic conditions without ruling them out, a process that requires extensive investigation steps not to mention the time needed to confirm persistence. Confirming clonality can bypass all these issues and can solve the problematic cases in which a neoplastic condition is co-existing with a secondary or congenital cause. Many methods are used to detect clonality but the most dependable is by performing cytogenetic, fluorescence in situ hybridization (FISH) and/or molecular tests. Abnormal immunophenotype and loss of X-linked polymorphism have many limitations in MPNs. It is currently widely accepted that detecting JAK2 mutation, particularly V617F mutation, is a major criterion in diagnosing myeloid neoplasms, especially MPNs. It is now incorporated in the WHO 2008, Nordic 2007, and BCSH 2007 classifications. ${ }^{3,35,36}$

\section{Phenotype and lineage determina- tion}

JAK2 abnormalities are seen in many types of hematologic neoplasms as mentioned earlier, and also are reported in $70-80 \%$ of cases with unexplained hepatic venous thrombosis

Table 1. Prevalence of JAK2 abnormalities in hematologic neoplasms.

\begin{tabular}{lcc}
\hline Neoplasms & JAK2 V617F mutation & Other JAK2 abnormalities \\
Polycythemia vera & $>95 \%$ & $4 \% J A K 2$ exon12 mutation \\
Essential thrombocythemia & $50-60 \%$ & \\
\hline Idiopathic myelofibrosis & $40-50 \%$ & \\
Chronic neutrophilic leukemia & $20 \%$ & \\
\hline Chronic myelomonocytic leukemia & $3-13 \%$ & \\
Juvenile chronic myelomonocytic leukemia & $20 \%$ & \\
\hline Atypical chronic myeloid leukemia & $20 \%$ & JAK2 rearrangement, $\mathrm{t}(8 ; 9), \mathrm{t}(9 ; 12), \mathrm{t}(9 ; 22)$ \\
MPN/MDS-U & $12-20 \%$ & \\
\hline MPN/MDS (RARSt) & $50-70 \%$ & \\
Myelodysplastic syndrome (RAEB,-5q) & $1-7 \%$ & $J A K 2$ rearrangement, $\mathrm{t}(9 ; 12)$ \\
\hline Secondary AML & $4 \%$ & $J A K 2$ rearrangement, $\mathrm{t}(8 ; 9)$ \\
AML (M6) & Few reported cases & \\
\hline AML (M7) & $15 \%$ & $J A K 2$ T875N \\
Mastocytosis & $0-25 \%$ & $J A K 2$ rearrangement, $\mathrm{t}(8 ; 9), \mathrm{t}(9 ; 12)$ \\
\hline Eosinophilic neoplasms & $0-2 \%$ & \\
Chronic myelogenous leukemia & Rare reported cases & \\
\hline ALL & Non & JAK2 rearrangement, $\mathrm{t}(8 ; 9)$, \\
& & $\mathrm{t}(9 ; 12), J A K 2$ R683G and less \\
ALL in Down syndrome patients & Non & frequently other R683 point mutations \\
& & JAK2 R683G, IREED del, \\
& & \\
\hline
\end{tabular}

without overt neoplasms. ${ }^{37}$ Not only, V617F mutation alone is seen in a wide range of myeloid neoplasms. This fact has created much hypothesis regarding the pathophysiology of these neoplastic conditions to the extent that some authors suggest that some disorders are in fact phases of a single disease. Many factors possibly play a role (Figure 5).

\section{The type of abnormality}

Some abnormalities are only reported in myeloid neoplasms. These are JAK2 rearrangements to $B C R: \mathrm{t}(9 ; 22)$ and NF-E2: $\operatorname{der}(9) \mathrm{t}(9 ; 12), \mathrm{V} 617 \mathrm{~F}$ and T875N point mutations and exon 12 mutations. On the other hand, R683G and IREED del are reported in lymphoid disorders. While rearrangements to TEL/ETV6: $\mathrm{t}(9 ; 12)$ and PCM1: $\mathrm{t}(8 ; 9)$ are reported in both lineages. Some of these abnormalities are only reported in a single entity (Table 1).

\section{The targeted cell or receptor}

It is suggested that the phenotype is determined by the ability of the affected stem cells to differentiate into different lineages, and on the receptor affected by the mutation. For example, EP0, TMP, G-CSF or GM-CSF receptors are targeted to give PV, ET, PMF or chronic myelomonocytic leukemia, respectively. ${ }^{38}$ Another example is the identification of homozygous JAK2 V617F mutation in the endothelial cells in the vessels of PV patients with Budd-Chiari syndrome. ${ }^{39}$ 
Genetic background of the patient

Gender is one of the possible determinants, as ET tend to occur in females more than males, while the opposite is true of PV. Iron metabolism is another factor, as iron depletion will lead to ET phenotype rather than PV. The opposite picture will be seen in relation to EPO bioavailability. SNPs are another possible participant as rs10974944 is significantly seen in patients with PV if compared to ET. ${ }^{4,29,38}$

\section{Dosage effect}

This appears to be the most important factor, and it can be explained by different thresholds of JAK2 kinase activity at which variable receptors, cytokines and proteasomes need to react. This is supported by the reported results of homozygous $J A K 2 \mathrm{~V} 617 \mathrm{~F}$ mutation occurring in $25-30 \%$ PV compared to $2-4 \%$ in ET. Few cases are reported that demonstrate dosage dependent phenotype, like those transforming from ET to PV with increasing JAK2 V617F mutation burden, or from PMF to PV with burden reduction after treatment with hydroxyurea (HU).$^{40}$ We have also encountered a case of PMF that transformed to ET after treatment with $\mathrm{HU}$ (K Alkhairi, KM Alayed, MK Alabdulaali, unpublished data, 2007) By monitoring allele burden it is found that $J A K 2 \mathrm{~V} 617 \mathrm{~F}$ mutation burden is increasing significantly from ET, PV to PMF. ${ }^{6,38,40-44}$ The levels at which the burden is found seem to correspond to a specific phenotype of $25 \%, 55 \%, 50 \%$ and $60 \%$ for ET, PV, PMF and post ET or PV myelofibrosis, respectively. ${ }^{43}$ Hypermethylation of the SOCS3 promoter was identified in nearly $1 / 3$ of patients with myelofibrosis and in both JAK2 mutation positive and negative cases ${ }^{27}$ but not in other types of MPNs; this again will strengthen the role of dosage effect.

\section{Pre-JAK2 mutation event}

Several findings raise the possibility of a pre- JAK2 mutation event leading or participating in the development of neoplasia: the presence of JAK2 mutation negative MPNs with $25 \%$ abnormal cytogenetics, the finding that $50 \%$ of JAK2 mutation positive cases are seen with recurrent cytogenetic abnormalities, and more interestingly, that the majority of JAK2 mutation positive cases develop JAK2 mutation negative leukemic blasts when they progress to AML. ${ }^{4,638,45}$ Some of the cytogenetic abnormalities are suspected to present more in association with JAK2 mutation to develop a specific phenotype like del $20 \mathrm{q}$ in cases with $\mathrm{PV}^{4}{ }^{4}$

\section{Clinical severity and progression}

Thrombosis, bleeding, splenomegaly, bone marrow failure and evolution to acute leukemia are the main complications of MPNs. In ET, many studies suggested that JAK2 mutation positive cases have a higher risk of developing venous thrombosis compared to patients

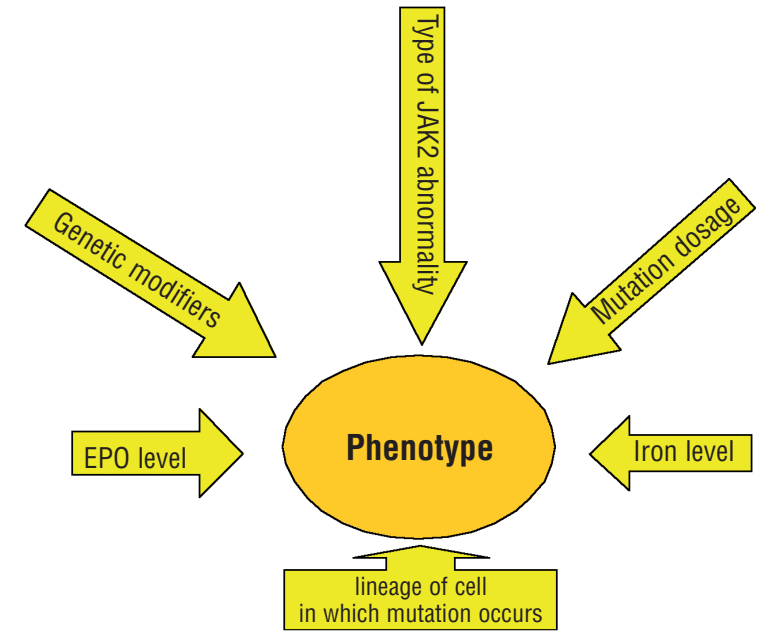

Figure 5. Factors that might determine the phenotype of JAK2 mutation positive disorders.

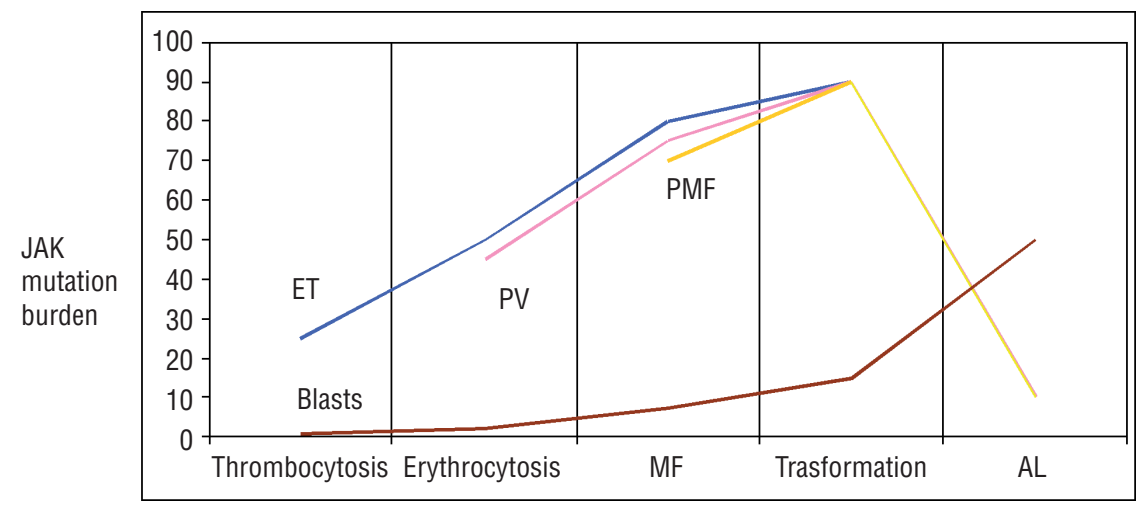

Figure 6. JAK2 mutation burden during progression of MPNs. AL: acute leukemia, ET: essential thrombocythemia, MF: myelofibrosis, PMF: primary myelofibrosis, PV: polycythemia vera.

with wild-type $J A K 2$. In a systematic literature review, Panayiotis D. Ziakas analyzed 17 studies with 2,905 ET patients, of whom 1,646 (65.7\%) patients were JAK2 V617F mutation positive. Thrombotic events occurred in 523 (31.8\%) of mutation positive cases and in 255 (20.3\%) of patients with wild-type JAK2. Finally, he concluded that JAK2 V617F patients have a 2-fold risk of developing thrombosis (OR 1.84, 95\%CI 1.40-2.43). ${ }^{46}$ Presence of inherited thrombophilia can increase the realtive risk of the development of thrombosis in patients $<60$ years of age with ET and JAK2 V617F mutation from $2.23(95 \% \mathrm{CI} 1.57-3.18)$ to 7.66 (95\%CI 2.66-22.03). ${ }^{47}$ High mutation dosage are significantly associated with larger spleen size, higher WBC count, higher lactate dehydogenase (LDH) level and higher risk of developing venous thrombosis or cardiovascular events in patients with ET or PV. However, these findings are not sufficient for chemical cytoreduction intended risk stratification which is still dependent on age, prior thrombotic event and cardiovascular event-related risk factors. ${ }^{2,441,48}$

It is still debatable whether an increased JAK2 mutation burden is associated with increased risk of evolution to acute leukemia. However, there is good evidence of its association with progression from ET to PV to PMF. If evolution to leukemia then occurs, in the majority of cases the leukemic cells will be JAK2 mutation negative as mentioned earlier. The overwhelming blasts will, therefore, reduce the allele mutation burden which might be considered a sign of evolution if it is not related to chemical cytoreductive therapy (Figure 6). ${ }^{41,44}$

\section{JAK2 as a therapeutic target}

For decades, the treatment options for patients with MPNs was limited to palliation and preventive measures against the development of thrombosis using aspirin, phlebotomy, splenectomy, splenic radiation or the use of steroids, androgens or EPO in patients with PMF. ${ }^{1}$ Since the middle of the $20^{\text {th }}$ century, aims are widening and touching the areas of prevention of leukemic transformation and cure with the use of cytoreductive medications like busulfan, HU and interferon-alpha (INF- $\alpha$ ), 
together with the trials of stem cell transplantation. But the use of these options always carries the risk of leukemogenesis. So, given the fact that MPNs are very slow progressing disorders and most of the patients are kept under strict control with non-chemical approaches, the use of these options is limited to high-risk patients.

In the last decade, the use of tyrosine kinase inhibitors is expanding in the management of hematologic neoplasms, especially after the successful results of imatinib in CML patients. However, the use of imatinib in MPNs is only achieving limited benefits. It is reported that the use of oral imatinib in PV can reduce the need for phlebotomy and spleen size, while parenteral administration can achieve remission in $22 \%$ of cases. ${ }^{1,449}$ Currently, there are many trials of new agents on patients with PMF, including farnesyl transferase, the aurora family of serine/threonine kinases, vascular endothelial growth factor (VEGF) tyrosine kinase, proteasome and fibrogenesis inhibitors, VEGF neutralizing antibodies and GX15-070MS, an antagonist of the BH3-binding groove of the Bcl-2 family. ${ }^{1,4}$

Many specific JAK2 inhibitors, like INCB018424, TG101209, TG101348, XL019 and TG10134841, are currently in phase I/II trials on advanced stages of MPNs I such as PMF, post-ET or PV myelofibrosis. Recent reports from the trials with INCB018424 are showing significant improvement in splenomegaly, constitutional symptoms, control of myeloproliferation and reduction in allele burden. Non-specific JAK2 inhibitors are also in clinical trials. These include: CEP-701 (an FLT3 inhibitor), tipifarnib (a farnesyltransferase inhibitor), ITF2357 (an HDAC inhibitor) and hypomethylating agents. The main drawbacks of JAK2 inhibitors are hematologic toxicity like neutropenia and thrombocytopenia, non-hematologic side effects which are mainly immunological and endocrinological. Beside that, currently used therapeutic options are well tolerated, and pegylated INF- $\alpha$ and parenteral imatinib are reported to achieve $18-22 \%$ remission rates, respectively. Another unpromising issue is the limitation in curing the disease or preventing evolution in the presence of a pre$J A K 2$ mutation event and JAK2 mutation negative leukemic transformation. The development of resistance in vitro is another worrying issue. ${ }^{1,4,648-50}$ For the anticipated toxic effects of JAK2 inhibitors, few agents targeting component downstream of JAK2 are currently investigated like those inhibiting BCL-XL or SOCS1 mimetics. ${ }^{1}$

\section{Conclusions}

$J A K 2$ abnormalities are important contributors but not the sole events in the development of hematologic neoplasms particularly MPNs. Its mutations, rearrangements or del are important to confirm clonality, and their type and dosage can help in classifying hematologic neoplasms. Currently, JAK2 abnormalities are mainly utilized in confirming clonality in diagnosing classical $B C R / A B L$ negative myeloproliferative neoplasms; however, we believe that their role will expand to be added in more hematologic neoplasms, to include the type of $J A K 2$ abnormality in the sub-classifications criteria and, possibly, to consider JAK2 mutation burden in the process of classification and in the assessment of transformation.

The JAK2 V617F positive MPNs are more closely related and appear with variable clinico-pathological phenotypes in response to different modifiers. They seem to have a more severe clinical course but there is still not sufficient available data to include this mutation in the risk stratification.

JAK2/STAT pathway is an important target for new therapeutics, but more studies are needed to minimize their toxicity.

\section{References}

1. Mughal TI, Goldman JM. Chronic myeloproliferative Disorders. 2008; Informa, UK.

2. Tefferi A. Classification, diagnosis and management of myeloproliferative disorders in the JAK2V617F Era. Hematology 2006:240-5.

3. Steven HS, Campo E, Harris NL, et al. WHO Classification of Tumors of Haematopoietic and Lymphoid Tissues. 2008; IARC, Lyon.

4. Silver RT, Tefferi A. Myeloproliferative disorders: biology and management. 2008; Informa Healthcare, USA.

5. Harmening DM. Clinical Hematology and fundamentals of hemostasis. 4th edition, 2002; F. A. Davis.

6. Levine RL, Gilliland G. Myeloproliferative disorders. Blood 2008; 112:2190-8.

7. Janus, Shorter Oxford English Dictionary. Vol 1, 3rd edition. Oxford University Press. 1973.

8. Porcu M, Gielen 0, Cools J, et al. JAK1 mutation analysis in T-ALL cell lines. Haematologica 2009;94:435-7.

9. De Vita S, Mulligan C, Mcelwaine S, et al. Loss-of-function JAK3 mutations in TMD and AMKL of Down syndrome. Br J Haematol 2007;137:337-41.

10. Kiyoi H, Yamaji S, Kojima S, Naoe T. JAK3 mutations occur in acute megakaryoblastic leukemia both in Down syndrome children and non-Down syndrome adults. Leukemia 2007; 21:574-6.

11. Sato T, Toki T, Kanezaki R, et al. Functional analysis of JAK3 mutations in transient myeloproliferative disorder and acute megakaryoblastic leukaemia accompanying Down syndrome. $\mathrm{Br} \mathrm{J}$ Haematol 2008;141:681-8.

12. Stoiber D, Kovacic B, Schuster C, et al. TYK2 is a key regulator of the surveillance of B lymphoid tumors. J Clin Invest 2004;114:1650-8.

13. Pritchard MA, Baker E, Callen DF, et al. Two members of the JAK family of protein tyrosine kinases map to chromosomes 1p31.3 and 9p24. Mammalian Genome 1992;3:36-8.

14. Saltzman A, Stone M, Franks C, et al. Cloning and characterization of human Jak-2 kinase: high mRNA expression in immune cells and muscle tissue. Biochem. Biophys. Res. Commun 1998;246:627-33.

15. Argetsinger LS, Campbell GS, Yang X, et al. Identification of JAK2 as a growth hormone receptor-associated tyrosine kinase. Cell 1993; 74:237-44.

16. Witthuhn BA, Quelle FW, Silvennoinen 0, et al. JAK2 associates with the erythropoietin receptor and is tyrosine phosphorylated and activated following stimulation with erythropoietin. Cell 1993;74:227-36.

17. Campbell GS, Argetsinger LS, Ihle JN, et al. Activation of JAK2 tyrosine kinase by prolactin receptors in $\mathrm{Nb} 2$ cells and mouse mammary gland explants. Proc Nat Acad Sci USA 1994;91:5232-6

18. Parganas E, Wang D, Stravopodis D, et al. Jak2 is essential for signaling through a variety of cytokine receptors. Cell 1998; 93:385-95.

19. Kralovics R, Passamonti F, Buser AS, et al. A gain-of-function mutation of JAK2 in myeloproliferative disorders. N. Engl J Med 2005;352:1779-90.

20. James C, Ugo V, Le Couedic JP, et al. A unique clonal JAK2 mutation leading to constitutive signalling causes polycythaemia vera. Nature 2005;434:1144-8.

21. Lacronique V, Boureux A, Della Valle V, et al. A TEL-JAK2 fusion protein with constitutive kinase activity in human leukemia. Science 1997;278:1309-12.

22. Peeters P, Raynaud SD, Cools J, et al. Fusion of TEL, the ETS-variant gene 6 (ETV6), to the receptor-associated kinase JAK2 as a result of $\mathrm{t}(9 ; 12)$ in a lymphoid and $\mathrm{t}(9 ; 15 ; 12)$ in a myeloid leukemia. Blood 1997;90:2535-40.

23. Malinge S, Ben-Abdelali R, Settegrana $C$, et al. Novel activating JAK2 mutation in a patient with Down syndrome and B-cell precursor acute lymphoblastic leukemia. Blood 2007;109:2202-4.

24. Kearney L, Gonzalez De Castro D, Yeung J, et al. Specific JAK2 mutation (JAK2R683) and multiple gene deletions in Down syndrome acute lymphoblastic leukemia. Blood 2009;113:646-8 
25. Mullighan CG, Zhang J, Harvey RC, et al. JAK mutations in high-risk childhood acute lymphoblastic leukemia. Proc Natl Acad Sci USA 2009;106:9414-8.

26. Elliott J, Suessmuth Y, Scott LM, et al. SOCS3 tyrosine phosphorylation as a potential bio-marker for myeloproliferative neoplasms associated with mutant JAK2 kinases. Haematologica 2009;94:576-80.

27. Fourouclas N, Li J, Gilby DC, et al. Methylation of the suppressor of cytokine signaling 3 gene (SOCS3) in myeloproliferative disorders. Haematologica 2008; 93:1635-44.

28. Landgren 0, Goldin LR, Kristinsson SY, et al. Increased risks of polycythemia vera, essential thrombocythemia, and myelofibrosis among 24577 first-degree relatives of 11039 patients with myeloproliferative neoplasms in Sweden. Blood 2008; 112: 2199-204.

29. Olcaydu D, Harutyunyan A, Jäger R, et al. A common JAK2 haplotype confers susceptibility to myeloproliferative neoplasms. Nature Genetics 2009; 41:450-4.

30. Conchon MR, Costa JL, Novaes MM, et al. Simultaneous detection of JAK2 V617F mutation and Bcr-Abl translocation in a patient with chronic myelogenous leukemia. Int J Hematol 2008;88:243-5.

31. McLornan D, Percy M, McMullin MF. JAK2 V617F: a single mutation in the myeloproliferative group of disorders. Ulster Med J 2006;75:112-9.

32. Hellström-Lindberg E, Cazzola M. The role of JAK2 mutations in RARS and other MDS. Hematology 2008;52-9.

33. Horn T, Kremer M, Dechow T, et al. Detection of the activating JAK2 V617F mutation in paraffin-embedded trephine bone marrow biopsies of patients with chronic myeloproliferative diseases. J Mol Diagn 2006;8:299-304.

34. Lippert E, Girodon F, Hammond E, et al. Concordance of assays designed for the quantification of JAK2V617F: a multicenter study. Haematologica 2009;94:38-45.

35. Odense HH, et al. Guidelines for the diagnosis and treatment of patients with polycythemia vera, essential thrombocythemia and idiopathic myelofibrosis. Nordic MPD Study Group, 2007.

36. McMullin MF, Bareford D, Campbell P, et al. Guidelines for the diagnosis, investigation and management of polycythaemia /erythrocytosis. Br J Haematol 2005;130: 174-95.

37. Goulding C, Uttenthal B, Foroni L, et al. The JAK2(V617F) tyrosine kinase mutation identifies clinically latent myeloproliferative disorders in patients presenting with hepatic or portal vein thrombosis. Int J Lab Hematol 2008;30:415-9.

38. Chloé J. The JAK2V617F mutation in polycythemia Vera and other myeloproliferative disorders: one mutation for three diseases? Hematology 2008;69-75.

39. Sozer S, Fiel MI, Schiano T, et al. The presence of JAK2V617F mutation in the liver endothelial cells of patients with BuddChiari syndrome. Blood 2009;113:5246-9.

40. Spivak JL, Silver RT. The revised World Health Organization diagnostic criteria for polycythemia vera, essential thrombocytosis, and primary myelofibrosis: an alternative proposal. Blood 2008;112:231-9.

41. Vannucchi AM, Antonioli E, Guglielmelli P, et al. Clinical profile of homozygous JAK2 $617 \mathrm{~V}>\mathrm{F}$ mutation in patients with polycythemia vera or essential thrombo- cythemia. Blood 2007;110:840-6.

42. Stauffer Larsen T, Pallisgaard N, Boe Møller M, et al. The JAK2 V617F allele burden in essential thrombocythemia, polycythemia vera and primary myelofibrosis impact on disease phenotype. Eur $\mathrm{J}$ of Haematol 2007;79:508-15.

43. Antonioli E, Guglielmelli P, Poli G, et al. Influence of JAK2V617F allele burden on phenotype in essential thrombocythemia. Haematologica 2008; 93:41-8.

44. Passamonti F, Rumi E. Clinical relevance of JAK2 (V617F) mutant allele burden. Haematologica 2009;94:7-10

45. Schaub FX, Jäger R, Looser R, et al. Clonal analysis of deletions on chromosome $20 \mathrm{q}$ and JAK2-V617F in MPD suggests that del $20 \mathrm{q}$ acts independently and is not one of the predisposing mutations for JAK2V617F. Blood 2009;113:2022-7.

46. Ziakas PD. Effect of JAK2 V617F on thrombotic risk in patients with essential thrombocythemia: measuring the uncertain. Haematologica 2008;93:1412-4.

47. De Stefano V, Za T, Rossi E, et al. Influence of the JAK2 V617F mutation and inherited thrombophilia on the thrombotic risk among patients with essential thrombocythemia. Haematologica 2009;94:733-7.

48. Vannucchi AM, Guglielmelli P, Pieri L, et al. Treatment options for essential thrombocythemia and polycythemia vera. Expert Rev Hematol 2009;2:41-55.

49. Rambaldi A, Barbui T, Barosi G. From palliation to epigenetic therapy in myelofibrosis. Hematology 2008: 83-91

50. Levine RL, Heaney M. New advances in the pathogenesis and therapy of essential thrombocythemia. Hematology 2008:76-82. 